\title{
RETORNO ÀS ATIVIDADES OCUPACIONAIS E SEXUAIS APÓS CIRURGIA DE REVASCULARIZAÇÃO DO MIOCÁRDIO
}

Dantas RAS, Aguillar OM, Barbeira CBS. Retorno às atividades ocupacionais e sexuais após cirurgia de revascularização do miocárdio. Rev Latino-am Enfermagem 2001 julho; 9(4):26-31.

Através de estudo prospectivo e longitudinal acompanhamos o retorno ao trabalho e às atividades sexuais de 17 homens (35 a 73 anos) após revascularização do miocárdio. Dados foram coletados através de entrevistas e transcritos em notas de campo desde a internação até 0 sexto mês após a alta. Dos 14 pacientes que relataram atividades ocupacionais e sexuais antes da cirurgia, apenas 8 retornaram ao trabalho $(p=0,016)$ e 10 à vida sexual $(p=0,0625)$. Concluindo, houve mudança de atitude para o não retorno ao trabalho e tendência ao não retorno à vida sexual na população estudada.

PALAVRAS CHAVE: revascularização miocárdica, trabalho, sexualidade

\section{RETURN TO OCCUPATIONAL AND SEXUAL ACTIVITY AFTER CORONARY ARTERY BYPASS SURGERY}

A prospective and longitudinal design was used to follow up the return to occupational and sexual activity among 17 males after coronary bypass surgery. The subjects' ages ranged from 35 to 73 years. Data collection was performed by using open interviews which were recorded in field notes during hospitalization after heart surgery and during 6 months following hospital discharge. Of the 14 patients who reported work and sexual activity before surgery, 8 were working again $(p=0.016)$, and 10 had returned to sexual activity $(p=0.0625)$. There was change in the subjects' attitudes leading to not returning to work and tendency to not resuming sexual activity in this population.

KEY WORDS: myocardial revascularization, sexuality, work

\section{REGRESO A LAS ACTIVIDADES OCUPACIONALES Y SEXUALES DESPUÉS DE CIRUGÍA DE REVASCULARIZACIÓN DEL MIOCÁRDIO}

A través de un estudio prospectivo y longitudinal, los autores siguieron el retorno al trabajo y a las actividades sexuales de 17 hombres (35 a 73 años) después de la revascularización del miocárdio. Los datos fueron recolectados a través de entrevista y transcritos en las notas de campo desde la hospitalización hasta el sexto mes después de la dada de alta. De los 14 pacientes que relataron actividades ocupacionales y sexuales antes de la cirugía, solamente 8 retornaron al trabajo $(p=0.016)$ y 10 a la vida sexual $(p=0.0625)$. Concluyendo, en la población estudiada hubo un cambio de actitud para el no regreso al trabajo y una tendencia a no regresar a la vida sexual.

PALABRAS CLAVES: revascularización del miocárdio, sexualidad, trabajo

\footnotetext{
${ }^{1}$ Professor Doutor, e-mail: rsdantas@eerp.usp.br, Escola de Enfermagem de Ribeirão Preto da Universidade de São Paulo, Centro Colaborador da OMS para 0 desenvolvimento da pesquisa em enfermagem
} 


\section{INTRODUÇÃO}

$N_{\text {o nosso dia a dia como enfermeiros, cada vez mais nos }}$ defrontamos com pessoas acometidas por doenças cardiovasculares, entre elas a doença arterial coronariana. Esta doença se manifesta na forma clínica de infarto agudo do miocárdio, angina e miocardiopatias e afeta parcela importante da população que se encontra economicamente ativa. De acordo com estudo em pacientes norte-americanos, cerca de $80 \%$ das pessoas acometidas retornam aos seus antigos empregos ${ }^{(1)}$.

As variáveis estudadas nas pesquisas que abordam 0 retorno ao trabalho após eventos cardíacos podem ser categorizadas em: demográficas (idade, sexo, situação sócio-econômica, estado civil e tipo de profissão), psico-sociais (depressão, apoio social, percepção do paciente com relação à sua capacidade de trabalho e sua auto-eficiência) e aquelas relacionadas ao ambiente de trabalho. Também têm sido abordados os fatores clínicos (gravidade da doença, dor, capacidade funcional e influências do tratamento tais como restrições médicas e efeitos de medicamentos). No entanto, nenhuma destas variáveis tem previsto com sucesso o retorno ao trabalho. De todos os fatores acima citados, aqueles relacionados à atual ocupação do indivíduo ou seu ambiente de trabalho são os que têm recebido menor atenção. Eles incluem satisfação com o emprego, demanda física e tensões da profissão ${ }^{(2)}$.

Vem sendo constatado que trabalhadores do sexo masculino, com profissões mais intelectuais e com alto status sócioeconômico retornam ao trabalho com maior freqüência que os trabalhadores braçais e as pacientes do sexo feminino ${ }^{(3-6)}$. Indivíduos com maior apoio social retornam ao trabalho mais rapidamente que aqueles que tiveram menor apoio. Por outro lado, fatores como obrigações familiares e compromissos financeiros podem forçar 0 retorno mais precoce ao trabalho ${ }^{(7)}$.

O retorno às atividades sexuais após eventos cardiológicos também tem sido objeto de estudo. Uma ampla proporção de pacientes não retornam à atividade sexual após a ocorrência da doença. Muitos fatores, incluindo as mudanças fisiológicas já esperadas e decorrentes do processo de envelhecimento, disfunções induzidas por medicamentos e alterações vasculares associadas com fatores de risco (por exemplo, diabetes, hipertensão e dislipidemias), somadas ao impacto emocional da doença cardíaca, podem influenciar a vida sexual destes indivíduos. Estes fatores podem explicar, ao menos parcialmente, a elevada prevalência de disfunção sexual entre pessoas acometidas por doença arterial coronariana ${ }^{(5,8)}$.

0 objetivo desta pesquisa foi investigar 0 retorno às atividades ocupacionais e sexuais durante os seis primeiros meses após a cirurgia de revascularização do miocárdio (RVM). Este estudo fez parte da uma investigação mais ampla que abordou o processo de reabilitação de pacientes submetidos a um protocolo de assistência de enfermagem para indivíduos revascularizados atendidos em um hospital de ensino brasileiro. Tal pesquisa também avaliou os problemas apresentados após a alta hospitalar, uso de medicamentos, controle dos fatores de risco coronarianos e adesão à um programa diário de caminhada ${ }^{(9)}$

\section{METODOLOGIA}

Local e design do estudo: para acompanhar o retorno ao trabalho e à vida sexual optamos por um estudo do tipo prospectivo e longitudinal que foi desenvolvido no Hospital das Clínicas de Ribeirão Preto, estado de São Paulo.

População e amostra: a população constituiu-se de pacientes que foram submetidos à cirurgia de RVM entre os meses de janeiro e maio de 1998. Sujeitos eram considerados inelegíveis para iniciar o estudo baseados nos seguintes critérios de exclusão: a RVM ter sido realizada concomitante com outra cirurgia cardíaca e ter inabilidade para comunicar-se devido a problemas físicos ou emocionais. Durante o período do estudo, 38 pacientes se enquadraram no estudo. Destes pacientes, 6 receberam alta antes de serem contactados pela enfermeira pesquisadora, 4 morreram durante a hospitalização, 3 tiveram complicações pós-cirúrgicas que impediram suas participações no protocolo proposto e 1 tinha deficiência auditiva. Assim, nossa amostra se constituiu de 24 sujeitos, sendo 23 homens. Durante o período de 6 meses de acompanhamento, 6 deles não retornaram às consultas ambulatoriais e tiveram seu processo de acompanhamento interrompido. No final de dezembro de 1998, finalizamos nosso protocolo com 18 pacientes. Como foi possível acompanhar o processo de reabilitação de apenas uma paciente do sexo feminino, decidimos excluí-la da análise final do estudo. Assim, o grupo estudado constitui-se de 17 homens, com idade entre 35 a 73 anos (média de $56 \pm 10,9$ anos), sendo 15 (88,2\%) casados, 14 (82,3\%) com primeiro grau incompleto e $16(94,1 \%)$ com renda familiar mensal menor que seis salários mínimos. Quatorze $(82,3 \%)$ desenvolviam atividades ocupacionais remuneradas antes da cirurgia. Quanto aos aspectos clínicos-cirúrgicos, dois terços (70,6\%) da amostra tinha infarto do miocárdio já diagnosticado, 52,9\% receberam enxertos mistos e tiveram entre $2(23,5 \%)$ e 3 pontes colocadas $(47,0 \%)$.

Proteção dos sujeitos envolvidos no estudo: Primeiramente, o projeto de pesquisa foi aprovado pelo Comitê de Ética da instituição onde se desenvolveu o estudo. Os objetivos do mesmo eram apresentados aos pacientes e familiares durante o período de internação pós-operatória. Com a concordância dos participantes, 0 termo de consentimento pós-esclarecido era assinado pelos envolvidos ${ }^{(10)}$. 
Procedimentos realizados: durante o estudo, os 17 participantes foram seguidos, pela enfermeira pesquisadora, na fase de hospitalização pós-operatória e durante os seis primeiros meses após a alta. Receberam informações orais e por escrito sobre a doença coronariana, o tratamento cirúrgico e mudanças no estilo de vida visando a promoção da saúde durante a internação e nos retornos ambulatoriais. Os aspectos vivenciados por eles no processo de reabilitação e no retorno às atividades eram discutidos. Esposas e outros membros da família eram encorajados a participar dos encontros, visando auxiliar no processo de resolução dos problemas apresentados, desenvolver conhecimentos e habilidades e prover apoio familiar ao doente.

Coleta dos dados: através de entrevistas abertas foram coletados os dados referentes ao processo de reabilitação cardíaca e relacionados ao retorno ao trabalho e à vida sexual, participação da família em sua recuperação e mudanças no estilo de vida. Para o registro dos dados foi utilizada a técnica de "notas de campo" ${ }^{\text {"(11) }}$. Nas notas de campo eram registrados os dados obtidos através da observação participante da pesquisadora nas consultas médicas ambulatoriais e dos diálogos ocorridos entre a enfermeirapesquisadora e os participantes. Não foi estipulado um roteiro prédeterminado para os encontros nos ambulatórios uma vez que os aspectos abordados nestas ocasiões eram aqueles trazidos pelos doentes e familiares e que diziam respeito ao processo de reabilitação após a cirurgia.

Análise dos dados: os dados foram analisados através de estatística descritiva e do uso do teste de MacNemar para pequenas freqüências esperadas (teste binomial aplicado à mudanças) para constatar associação entre tempo de reabilitação e o retorno às atividades ocupacionais e sexuais. 0 nível de significância considerado foi de $5 \%(\alpha=0,05)$.

\section{RESULTADOS}

Dos 17 participantes, 14 (82,3\%) desempenhavam atividades ocupacionais remuneradas antes da RVM. Tais atividades eram caracterizadas por serem não ou semi-qualificadas e por exigirem esforço físico de média ou alta intensidade para serem executadas. Cinco (35,7\% dos 14$)$ trabalhavam na lavoura, $4(28,5 \%)$ atuavam no comércio; $3(21,4 \%)$ na construção civil; $1(7,1 \%)$ era motorista de ônibus e o último $(7,1 \%)$ era porteiro. Destes 14 pacientes, $7(50 \%)$ já eram aposentados mas continuavam a desempenhar atividades remuneradas para aumentar a renda familiar.

A realização de atividade ocupacional remunerada antes e seis meses após a RVM está apresentada na Tabela 1. Constatamos que houve mudança de atitude na população estudada para o não retorno às atividades ocupacionais durante o período analisado $(p=$ $0,016)$. Seis meses após a cirurgia, $8(57,1 \%$ dos 14 e $47,0 \%$ do total de pacientes) haviam retornado ao trabalho sendo que 5 eram não aposentados com menos de 65 anos de idade, 2 aposentados com mais de 65 anos e 1 aposentado com menos de 65 anos. Em geral, o retorno ocorreu entre o terceiro e o quarto mês de reabilitação.

Tabela 1 - Distribuição dos 17 pacientes revascularizados segundo realização de atividades ocupacionais remuneradas desenvolvidas antes e seis meses após revascularização do miocárdio (RVM)

\begin{tabular}{lcccc}
\hline $\begin{array}{l}\text { Realização de atividades } \\
\text { ocupacionais remuneradas }\end{array}$ & \multicolumn{5}{c}{ Antes da RVM Seis meses após RVH' } \\
& $\mathbf{n}^{\mathbf{0}}$ & $\%$ & $\mathbf{n}^{\mathbf{0}}$ & $\%$ \\
\hline Sim & 14 & 82,3 & 8 & 47,0 \\
Nẫo & 3 & 17,6 & 9 & 52,9 \\
\hline${ }^{*} p=0,016$ (teste de MacNemar) & & &
\end{tabular}

As causas alegadas para o não retorno às atividades ocupacionais foram: desejo da família para que deixassem de trabalhar após a cirurgia; não compensação financeira; opção por aumentar as atividades de lazer; não liberação médica e solicitação da aposentadoria.

O comportamento da realização de atividade sexual antes e seis meses após a cirurgia está apresentada na Tabela 2. Constatamos que houve uma tendência na população estudada de mudar sua atitude com relação às atividades sexuais, não retornando as mesmas após a RVM $(p=0,0625)$. Dos 17 participantes, $14(82,3 \%)$ alegaram vida sexual ativa antes da revascularização cardíaca. Destes, 10 (71,4\% dos 14 ou 58,9\% do total) haviam retornado às atividades sexuais ao término do acompanhamento. Quanto ao período em que se deu este retorno tivemos que $8(47,0 \%)$ pacientes reiniciaram às atividades sexuais dois meses após a RVM e 2 (11,8\%) no terceiro mês. Dos 4 pacientes $(28,5 \%$ dos 14$)$ que não haviam retornado à vida sexual, $2(14,2 \%)$ alegaram a não liberação médica e $2(14,2 \%)$ referiram perda da libido em decorrência dos problemas de saúde.

Tabela 2 - Distribuição dos 17 pacientes revascularizados segundo a realização de atividades sexuais antes e seis meses após revascularização do miocárdio (RVM)

\begin{tabular}{lcccc}
\hline $\begin{array}{l}\text { Realização de atividades } \\
\text { se xuais }\end{array}$ & \multicolumn{3}{c}{ Antes da RVH Seis meses após RVH' } \\
& $\mathbf{n}^{\mathbf{0}}$ & $\%$ & $\mathbf{n}^{\mathbf{0}}$ & $\%$ \\
\hline Sim & 14 & 82,3 & 10 & 58,9 \\
Nẫo & 3 & 17,6 & 7 & 41,1 \\
\hline${ }^{*} p=0,0625$ (teste de MacNemar) & & &
\end{tabular}


Os problemas, medos e inseguranças no retorno à vida sexual foram relatados por $2(14,2 \%)$ dos pacientes, os quais alegaram que as esposas estavam nervosas, comprometendo a vida sexual do casal. Por sua vez, as esposas também apresentavam incertezas, sendo que 5 delas $(35,7 \%)$ expuseram suas dúvidas durante os nossos encontros, questionando qual seria o melhor período para reiniciar estas atividades e se havia risco de novo episódio isquêmico. Salientaram o medo de prejudicarem a saúde dos maridos e a perda do desejo sexual em decorrência da situação gerada pela doença e cirurgia. Tais esposas nos relataram que, embora os maridos estivessem querendo reiniciar a vida sexual, elas sentiam-se inseguras pois não se consideravam orientadas sobre este aspecto.

\section{DISCUSSÃO}

Os nossos resultados referentes ao não retorno às atividades ocupacionais e sexuais seis meses após a RVM são pertinentes aos reportados em outros estudos ${ }^{(1,4-5,12)}$. Entretanto, em nossa investigação, 0 alto índice de não retorno ao trabalho pode ser explicado pelo fato de termos considerados também os pacientes já aposentados entre aqueles que desempenhavam atividades ocupacionais remuneradas por ocasião da cirurgia. Vem sendo demonstrado que pacientes idosos deixam seus trabalhos, mesmo na ausência de sérios problemas de saúde ${ }^{(5,12)}$. Outros motivos podem ter sido a baixa qualificação profissional dos participantes e a falta de um protocolo médico que uniformizasse os parâmetros clínicos para liberá-los para o trabalho. Tal situação já havia sido detectada entre pacientes brasileiros ${ }^{(4)}$.

A participação de pacientes em programas de reabilitação cardíaca, com aconselhamento profissional para controle dos fatores de risco e realização de exercícios físicos, venha sendo defendida como um dos aspectos positivos para garantir a capacidade de trabalho dos cardiopatas ${ }^{(12-13)}$. Entretanto, alguns estudos não conseguiram obter diferença, estatisticamente significante, quando compararam os níveis de retorno às atividades ocupacionais e sexuais dos pacientes que participaram destes programas com os níveis dos pacientes que haviam recebido o cuidado usual, ou seja, orientações não sistematizadas e ausência de programa de condicionamento físico $^{(1,7,14-15)}$.

O retorno ao trabalho depende de certas condições. Os pacientes podem não voltar às suas atividades quando os sintomas apresentados interferem em seu estilo de vida, quando cedem aos pedidos das esposas, receosas da volta ao trabalho ou quando desejam aumentar o tempo de recuperação pós operatória. Podem optar por não voltarem ao trabalho quando apresentam complicações, são super protegidos por estarem doentes ou quando desconhecem quais são as condições clínicas que caracterizam um comportamento saudável ${ }^{(16)}$.

A participação da família no não retorno ao trabalho pôde ser observada durante o nosso estudo. Outro estudo que abordou a influência de familiares e profissionais da saúde na recolocação social de indivíduos revascularizados constatou que, para $42,1 \%$ dos pacientes, o médico e a família exerceram um fator essencial para o não retorno ${ }^{(4)}$. A combinação de uma superproteção familiar dizendo ao paciente o que ele não deve fazer, associado com o médico falhando em instruir o que ele pode fazer resultará em uma prolongada invalidez e falha em reassumir suas atividades profissionais ${ }^{(17)}$.

Com relação a participação do enfermeiro influenciando o retorno dos pacientes ao trabalho, estudo realizado em hospitais públicos brasileiros, observou que tais profissionais e seus auxiliares foram substituídos por psicólogos, nutricionistas e fisioterapeutas nas condutas educativas direcionadas a estes pacientes ${ }^{(18)}$. Acreditamos que outros estudos devem ser desenvolvidos para melhor avaliar a atual participação do enfermeiro nessa atividade, não só em hospitais públicos mas também outros locais de saúde. Embora os pacientes acompanhados em nosso estudo tivessem o seguimento da enfermeira-pesquisadora, fornecendo orientações quanto ao retorno às atividades, controle dos fatores de risco e programa diário de caminhadas, não havia uma proposta formal para um acompanhamento multidisciplinar visando a reabilitação cardíaca dos pacientes atendidos por esta instituição. A abordagem multidisciplinar se faz necessária para satisfazer os enfoques multifatoriais da doença coronariana e do seu processo de reabilitação.

Quanto às atividades sexuais após cirurgia de RVM, nossos resultados demonstraram tendência ao não retorno à vida sexual na população estudada. O período em que se deu o retorno é coincidente com o fim da cicatrização da esternotomia, ou seja, em torno de 8 semanas após a cirurgia, e já havia sido constatado como sendo 0 mais freqüente para se reiniciar estas atividades ${ }^{(19)}$. Esta situação nos chamou a atenção uma vez que, neste período de 2 a 3 meses após a revascularização, os pacientes geralmente não haviam ainda sido avaliados em um segundo retorno médico. 0 primeiro retorno após a alta ocorreu cerca de 30 dias e o segundo retorno era marcado no terceiro mês de reabilitação pós-operatória, ou seja, eles reiniciaram tal atividade sem terem sido formalmente liberados pela equipe médica. Quando perguntávamos se haviam retornado às atividades sexuais e se estavam tendo algum tipo de problema todos referiram não terem queixas cardiovasculares ou de qualquer outro tipo. Esta situação nos chamou a atenção uma vez que tal assunto era pouco abordado nas consultas médicas e é sabido que pode haver prejuízo do desempenho sexual entre cardiopatas do sexo masculino e na faixa etária em que se encontravam a maior parte dos sujeitos estudados. 
Ressaltamos nossa constatação de que os aspectos relacionados à vida sexual foi pouco discutido entre o médicopaciente-esposa. Muitas vezes tal abordagem era sugerida pela enfermeira-pesquisadora, durante sua participação no retorno médico dos pacientes. Essa nossa observação é semelhante àquela relatada por outro autor brasileiro ${ }^{(18)}$ em seu estudo. Analisando os relatos de revascularizados acerca das categorias de informações fornecidas pelos profissionais de saúde, ele constatou que as referentes às atividades sexuais foram aquelas abordadas com menor freqüência que às relacionadas ao retorno ao trabalho e a prática de atividades esportivas.

Há inúmeros fatores psicosociais que podem influenciar quando e com que grau de conforto o paciente retorna às atividades sexuais. Após a revascularização do miocárdio ele está sujeito à depressão enquanto ainda está internado ou após retornar ao lar, 0 que irá afetar seu nível de energia e interesse em reassumir a vida sexual ${ }^{(19)}$. Esta depressão pode durar dias, semanas ou meses e a diminuição da libido, bem com a disfunção erétil ocasional podem ser vividas neste período. Estudo realizado ${ }^{(20)}$ constatou que, 15 meses após a RVM, 47\% dos pacientes reportaram não mudanças na vida sexual, quase a mesma proporção da soma dos que reportaram diminuição (36\%) e abstinência sexual (8\%). Outros estudos têm demonstrado uma significante proporção de homens referindo deteriorização da vida sexual após a cirurgia ${ }^{(21-22)}$.

Como mostrou nossos resultados, as esposas também podem se sentir emocionalmente abaladas, com receio de prejudicar

\section{REFERÊNCIAS BIBLIOGRÁFICAS}

1. Froelicher ES, Newton KM, Livingston M. Return to work, sexual activity, and other activities after acute myocardial infarction. Heart \& Lung 1994; 23(5):423-35.

2. Pravikoff DS. Return to work: factors and issues of vocational counseling. In: Wenger NK, Smith, LK, Froelicher, ES, Comoss, PM. Cardiac rehabilitation. A guide to practice in 21st century. New York: Marcel Dekker, 1999. p. 295-302.

3. Almeida D, Bradford JM, Wenger NK, King SB, Hurst JW. Return to work after coronary artery bypass surgery. Circulation 1983; 68 (Suppl.2):205-13.

4. Bittar OJNV. Retorno ao trabalho após revascularização do miocárdio. [tese]. Ribeirão Preto (SP): Faculdade de Medicina de Ribeirão Preto/USP; 1992.

5. Mark DB, Lam LC, Lee KL, Clapp-Channing NE, Williams RB, Pryor $\mathrm{DB}$ et al. Identification of patients with coronary disease at high risk for loss of employment. A prospective validation study. Circulation 1992; 68(5):1485-94.

6. Riegel BJ, Dracup KA. Does overprotection cause cardiac invalidism after acute myocardial infarction. Heart \& Lung; 21(6):529-35. a saúde do marido e agirem de modo a evitar o retorno à vida sexual. Podem, ainda, superproteger os companheiros ou se ressentir de seus novos encargos no lar. A combinação de todos estes fatores certamente contribuem para o detrimento da vida do doente mais que a própria doença coronariana ${ }^{(19)}$. Ao se discutir disfunção sexual com o paciente cardíaco, o enfoque está quase sempre na disfunção erétil masculina. No entanto, a interação entre o casal tem uma relação direta e intensa sobre o nível de atividade sexual a ser determinada e mantida. 0 estresse envolvido na doença cardíaca e/ou seu tratamento pode exacerbar problemas já existentes entre os parceiros $^{(23)}$.

\section{CONCLUSÃO}

Como conclusão nosso estudo aponta mudança desfavorável de atitude para o não retorno ao trabalho e à vida sexual na população estudada, por motivos variados. Outros fatores, além da doença coronariana, podem influenciar a conduta dos pacientes para não reassumirem estas atividades tais como idade avançada, influência da família, baixo grau de escolaridade e de qualificação profissional. Os pacientes que retornaram às atividades ocupacionais e sexuais o fizeram entre o segundo e terceiro mês após a cirurgia de revascularização do miocárdio. Esses parâmetros devem ser objetos de pesquisa para enfermeiros visando melhorar a assistência e o ensino de enfermagem nesta área.

7. Burgess AW, Lerner DJ, D'Agostino RB, Vokonas PS, Hartman $\mathrm{CR}$, Gaccione P. A randomized control trial of cardiac rehabilitation. Soc Sci Med; 24(4):359-70.

8. Jackson G, Betteridge J, Dean J, Hall R, Holdright D, Holmes S et al. A systematic approach to erectile dysfunction in the cardiovascular patient: a consensus statement. Int J Clin Pract 1999; 53(6):445-51. 9. Dantas RAS. Reabilitação de pacientes após cirurgia de revascularização do miocárdio. [tese]. Ribeirão Preto (SP): Escola de Enfermagem de Ribeirão Preto/USP; 1999.

10. Ministério da Saúde (BR). Resolução n.1, de 13 de junho de 1988. Normas de pesquisa em saúde. Brasília (DF): Ministério da Saúde; 1998. Cap.2, p. 4-7: Aspectos éticos da pesquisa em seres humanos. 11. Bogdan R, Biklen S. Investigação qualitativa em educação: uma introdução à teoria e aos métodos. Tradução de Maria João Alvarez, Sara Bahia dos Santos e Telmo Mourinho Baptista. Portugal: Porto Editora; 1994.

12. Mayou R, Bryant B. Quality of life after coronary artery surgery. $Q$ J Med 1987; 62(239):239-48.

13. Stern M, Pascale L, Ackerman A. Life adjustment post myocardial infarction: determining predictive. Arch Intern Med 1987; 137(12):1680-5. 
14. Engblom E et al. Cardiac rehabilitation and return to work after coronary artery bypass surgery. Qual Life Res 1994; 3(3):207-13.

15. Oldridge $\mathrm{N}$ et al. Effects of quality of life with comprehensive rehabilitation after acute myocardial infarction. Am J Cardiol 1991; 67(13):1084-9.

16. Tack BB, Gilliss CL. Nurse-monitored cardiac recovery: a description of the first 8 weeks. Heart \& Lung 1990; 19(5):492-9.

17. Frank KA, Heller SS, Kornfeld, DS. A survey of adjustment to cardiac surgery. Arch Intern Med 1972; 130(5):735-8.

18. Bittar OJNV. Recolocação social entre pacientes com revascularização do miocárdio: influências da família e de profissionais da saúde. Medicina/Ribeirão Preto 1994; 27(3/4):368-75.
19. Scalzi CC, Burke L.E. Sexual counseling. In: Underhill SL, Woods, SL, Froelicher, ES, Halpeny, CJ, editors. Cardiac Nursing. $2^{\text {a }}$ ed. Philadelphia: J.B. Lippincont, 1989. p. 757-65.

20. Papadopoulos C, Shelley SI, Piccolo M, Beamont C, Barnett L. Sexual activity after coronary bypass surgery. Chest 1986; 90(5):68185.

21. Sjöland H, Wiklund I, Caidahl K, Hartford M, Karlsson T, Herlitz J. Improvement in quality of life differs between women and men after coronary artery bypass surgery. J Int Med 1999; 245:445-54.

22. Westin RC, Carlsson R, Israelsson B, Willenheimer R, Cline C, McNeil EF. Quality of life in patients with ischaemic heart disease: a prospective controlled study. J Int Med 1997; 242:239-47.

23. Taylor HA Sexual activity and the cardiovascular patient: guidelines. Am J Cardiol 1999; 84(5B):6N-10N. 